\title{
THREE
}

\section{EARLY ENGLISH METRICAL}

\author{
ROMANCES.
}




\section{THREE}

\section{EARLY ENGLISH METRICAL ROMANCES.}

WITH AN

\section{INTRODUCTION AND GLOSSARY.}

EDITED FROM A MS. IN THE POSSESSION OF J. I, BLACKBURNE, ESQ. M.P. BY JOHN ROBSON, ESQ.

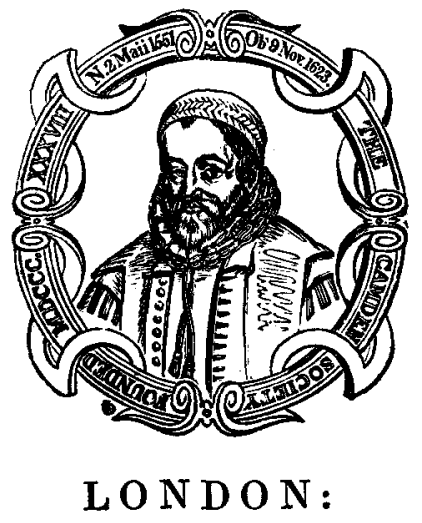

PRINTED FOR THE CAMDEN SOCIETY, BY JOHN BOWYER NICHOLS AND SON, PARLIAMENT STREET. M.DCCC.XLII. 


\section{COUNCIL}

$\mathbf{O F}$

\section{THE CAMDEN SOCIETY,}

FOR THE YEAR 1841-2.

\section{President,}

THE RIGHT HON, LORD FRANCIS EGERTON, M.P.

THOMAS AMYOT, ESQ. F.R.S. Treas. S.A. Director. THE RIGHT HON. LORD BRAYBROOKE, F.S.A. JOHN BRUCE, ESQ. F.S.A. Treasurer. JOHN PAYNE COLLIER, ESQ. F.S.A. C. PURTON COOPER, ESQ. Q.C., D.C.L., F.R.S., F.S.A. T. CROFTON CROKER, ESQ. F.S.A., M.R.I.A. SIR HENRY ELLIS, K.H., F.R.S., Sec. S.A. JAMES ORCHARD HALLIWELL, ESQ. F.R.S., F.S.A. THE REV. JOSEPH HUNTER, F.S.A. SIR FREDERICK MADDEN, K.H., F.R.S., F.S.A. JOHN GAGE ROKEWODE, ESQ. F.R.S., Dir. S.A. THOMAS STAPLETON, ESQ. F.S.A. WILLIAM J. THOMS, ESQ. F.S.A. Secretary. ALBERT WAY, ESQ. M.A., F.S.A. THOMAS WRIGHT, ESQ. M.A.. F.S.A. 